\title{
Heparin Inhibition of Ferredoxin-NADP Reductase in Chloroplast Thylakoid Membranes
}

\author{
JONATHAN P. HOSLER ${ }^{1}$ AND CHARLES F. YOCUM ${ }^{2}$ \\ Division of Biological Sciences and Department of Chemistry, The University of Michigan, \\ Ann Arbor, Michigan 48109 \\ Received June 6, 1984, and in revised form September 17, 1984
}

Heparin, an anionic polysaccharide, inhibited the ferredoxin-catalyzed reduction of NADP in spinach chloroplast thylakoid membranes. Under the same conditions of assay, heparin did not interfere markedly with photoreduction of methyl viologen, anthraquinone sulfonate, or ferredoxin. A kinetic analysis of the heparin-induced interference with NADP photoreduction showed partial competitive inhibition. Heparin also interfered with NADPH oxidation by membrane-bound ferredoxin-NADP reductase (with dichlorophenol-indophenol as the acceptor) by a mechanism that involves partial competitive inhibition. This reaction was sensitive to the presence of salts; increasing ionic strength increases the heparin $K_{i}$ for inhibition of NADPH oxidation. These results show that heparin binds to ferredoxin-NADP reductase, and in doing so interferes with binding to the reductase by both ferredoxin and NADP $(H)$. Since heparin is redox inactive and does not interfere with the photophosphorylation reaction, it is a useful inhibitor of thylakoid membrane reactions which require the catalytic activity of ferredoxin-NADP reductase. 1985 Academic Press, Inc.

Spinach ferredoxin-NADP reductase $(\mathrm{FNR})^{3}$ is a membrane-bound flavoprotein which catalyzes several two-electron transfer reactions, including ferredoxinmediated NADP reduction (1-3) and the reduction of various dyes by NADPII (diaphorase reaction) $(4,5)$. The flavoprotein contains separate binding sites for ferredoxin and NADP(H) (6); binary complexes of ferredoxin and FNR and of NADP and FNR have been shown to occur (7-9). Both of these complexes dissociate as the ionic strength of the medium is

\footnotetext{
${ }^{1}$ Present address: Department of Botany, Duke University, Durham, N. C. 20706.

2 To whom correspondence should be addressed.

abbreviations used: FNR, ferredoxin-NADP reductase; Fd, ferredoxin; Chl, chlorophyll; PS, photosystem; AQS, anthraquinone-2-sulfonate; Tricine, $\mathrm{N}$-[2-hydroxy -1,1-bis(hydroxymethyl)ethyl]glycine; Hepes, 4-(2-hydroxyethyl)-1-piperazineethanesulfonic acid; DCPIP, dichlorophenolindophenol; $\mathrm{MeV}$, methyl viologen.
}

increased (9), suggesting that ferredoxin and NADP(H) binding to FNR depends primarily upon ionic interactions.

Reversible inhibition has been demonstrated at both the ferredoxin and pyridine nucleotide binding sites of FNR. Competitive inhibition of NADP binding by 2 -AMP during a transhydrogenase reaction was demonstrated by Keister et al. (1), while inorganic pyrophosphate competitively inhibits ferredoxin binding during NADP photoreduction in isolated thylakoids, and inhibits the formation of the ferredoxin:FNR complex (10). Whole-chain electron transport from $\mathrm{H}_{2} \mathrm{O}$ to methyl viologen is unaffected by pyrophosphate, but the high concentration of pyrophosphate required to inhibit NADP reduction $\left(K_{i}=9.7 \mathrm{mM}\right)$ may be undesirable during assays of photosynthetic phosphorylation. We therefore sought an alternate inhibitor which would reversibly prevent ferredoxin binding to FNR. The use of a non-redox- 
active molecule is desirable in order to eliminate any competing electron transfer activity. One possibility would be the use of apoferredoxin, prepared by removing the $\mathrm{Fe} / \mathrm{S}$ center from the protein. This, however, is a costly procedure in terms of the amounts of native protein needed to prepare sufficient apoferredoxin for many experiments. Another approach would be to use a readily available, non-redox-active molecule with a molecular weight and ionic composition similar to ferredoxin. The polysaccharide heparin meets these qualifications.

Since heparin is composed of repeating dimers of glucosamine and glucuronic acid, and each dimer contains one carboxyl and two sulfate groups (11), the molecule is polyanionic at physiological $\mathrm{pH}$. Measurements of heparin molecular weights range from 6000 to 18,000 ; however, the value of $17,000 \mathrm{kDa}$ was chosen for concentration calculations in the experiments reported here based on the conclusions of Sumyk and Yocum (12). Heparin will not catalyze electron transfer; therefore, with some obvious limitations, heparin can be considered a non-redox-active ionic analog of ferredoxin. Our results show that heparin inhibits both ferredoxin and NADPH binding to membrane-bound FNR. The inhibitory heparin:FNR complex shares a number of properties with the ferredoxin:FNR complex.

\section{MATERIALS AND METHODS}

Spinach thylakoid membranes were prepared by the method of Robinson and Yocum (13). Ferredoxin was purified from spinach as described by Petering and Palmer (14), with the modifications of Yocum (15).

Assays of NADP photoreduction were performed in a reaction mixture containing $50 \mathrm{~mm}$ Tricine $(\mathrm{pH}$ 8.0 ), $50 \mathrm{~mm} \mathrm{NaCl}, 3 \mathrm{~mm} \mathrm{MgCl}, 5 \mathrm{~mm} \mathrm{NaH} \mathrm{PO}_{4}, 1$ mм ADP, $10 \mu \mathrm{M}$ ferredoxin (unless otherwise specified), $1 \mathrm{~mm}$ NADP, and $30 / 40 \mu \mathrm{g}$ Chl in a total volume of $1.5 \mathrm{ml}$. The rate of NADP reduction was measured by oxygen evelution in the presence of 840 units of catalase. This method was carefully calibrated to actual NADPH formation by measuring the concentration of photoreduced NADPH by oxidation with phenazine methosulfate. Two mole equivalents of NADP were reduced for every mole equivalent of oxygen evolved. Flestron transport to other PSI acceptors (ferredoxin/oxygen, AQS,
$\left.\mathrm{Fe}(\mathrm{CN})_{6}^{3-}, \mathrm{MeV}\right)$ was assayed in the reaction mixture described for NADP reduction, but without the addition of ferredoxin/NADP and catalase. Rates of oxygen evolution and uptake associated with the various electron transport reactions were measured with a Clark-type electrode fitted to a thermostatted $\left(25^{\circ} \mathrm{C}\right)$ cuvette. Saturating light $\left(10^{6} \mathrm{ergs}^{-2} \mathrm{~s}^{-1}\right)$ was provided by an Oriel Model 6235 light source filtered through $5 \mathrm{~cm}$ of an $0.2 \%$ solution of $\mathrm{CuSO}_{4}$ and a red cut-on filter $(>600 \mathrm{~nm})$.

The reduction of DCPIP by NADPH catalyzed by membrane-bound FNR was followed by the change in absorbance at $600 \mathrm{~nm}$ using an Aminco DW-2 spectrophotometer in the split-beam mode. Identical stirred cuvettes containing 20 mм Hepes- $\mathrm{NaOH}(\mathrm{pH}$ 7.5), $66 \mu \mathrm{M}$ DCPIP, and thylakoid membranes (13 $\mu \mathrm{g}$ $\mathrm{Ch} 1 / \mathrm{ml}$ ) were prepared; the reaction was initiated by the injection of a small volume of NADPH into one of the cuvettes. The ionic strength of the medium was adjusted by dilution of the buffer, or by addition of $\mathrm{NaCl}$. The slow rate of nonenzymatic reduction of DCPIP by NADPH was subtracted from all rates presented.

Chlorophyll was determined by the method of Arnon (16). Heparin, NADP, NADPH, ADP, catalase, Tricine, Hepes, and DCPIP were obtained from Sigma. Anthraquinone sulfonate was obtained from Eastman Organies and recrystallized; methyl viologen was purchased from Aldrich. All other chemicals used were of the purest grades commercially available.

\section{RESULTS}

Low concentrations of heparin effectively inhibit electron transport through FNR to NADP; NADP photoreduction, mediated by $10 \mu \mathrm{M}$ ferredoxin, was $60 \%$ inhibited by $20 \mu \mathrm{M}$ heparin (Fig. 1). Higher

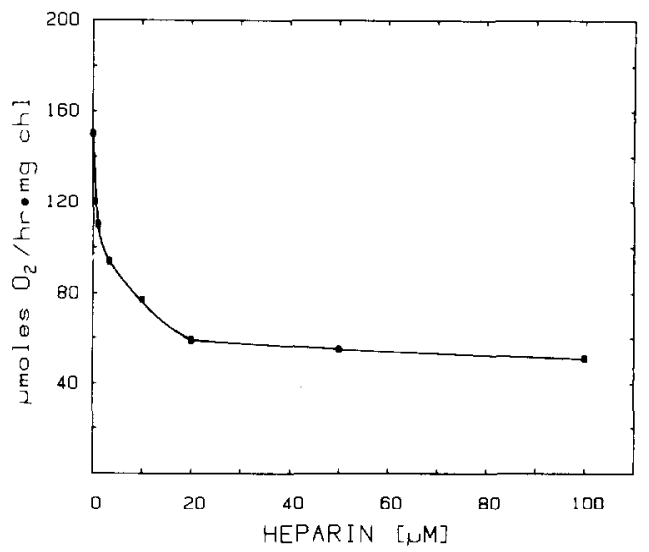

FIG. 1. Inhibition of photosynthetic NADP reduction by heparin. Assay conditions are described under Materials and Methods. 
concentrations of heparin $(100 \mu \mathrm{M})$ inhibited the rate of NADP reduction by $66 \%$, while ferredoxin-mediated oxygen reduction, catalyzed by $21 \mu \mathrm{M}$ ferredoxin in the absence of NADP, was only $9 \%$ inhibited by $10 \mu \mathrm{M}$ heparin, and $19 \%$ by $100 \mu \mathrm{M}$ heparin (Table I). This latter reaction presumably does not require FNR. Electron transport to other negatively charged PSI acceptors was only minimally affected by heparin; Table I shows that the rate of electron flow to AQS and $\mathrm{Fe}(\mathrm{CN})_{6}^{3-}$ is inhibited $9 \%$ by $10 \mu \mathrm{M}$ heparin. This slight inhibition may be due to ionic competition between negatively charged species (heparin and the acceptor) for the site of reduction at PSI. The addition of $100 \mu \mathrm{M}$ heparin had a greater effect on electron flow to ferredoxin/oxygen than it did when $\mathrm{AQS}^{-}$or $\mathrm{Fe}(\mathrm{CN})_{6}^{3-}$ were used as acceptors (Table I). The higher molecular weight of ferredoxin may account for this greater sensitivity. Electron transport to the cationic acceptor $\mathrm{MeV}$ was unaffected by 10 $\mu \mathrm{M}$ heparin, and was inhibited less than $5 \%$ by $100 \mu \mathrm{M}$ heparin (Table I). This observation supports the hypothesis of a weak ionic competition between heparin and negatively charged electron acceptors, and also provides strong evidence that the membrane-bound components of the electron transport chain prior to FNR are insensitive to inhibition by heparin.

Double-reciprocal plots were used to analyze the inhibition exerted by heparin on N $\Lambda D P$ reduction. A plot of $1 /$ ferre- doxin] vs $1 / v$ in the absence and presence of varying concentrations of heparin produced a family of lines which intersect on the $1 / v$ axis, suggesting a competitive interaction between ferredoxin and heparin (Fig. 2a). These results are best explained by heparin inhibition of ferredoxin binding to FNR. Dixon plots of these data ( $1 / v$ vs heparin concentration) were nonlinear (Fig. 2b), indicating that heparin inhibition of ferredoxin binding to FNR was of a partial competitive nature (17). Thus, while heparin interferes with binding of ferredoxin to FNR, both native and inhibited reductase are able to mediate electron flow from ferredoxin to NADP. The inhibited reductase, however, catalyzed the reaction at a slower rate. The $K_{i}$ for the heparin:FNR interaction (determined following (18)) was $0.3 \mu \mathrm{M}$, while the $K_{m}$ for ferredoxin in this reaction was measured to be $4.0 \mu \mathrm{M}$.

Doubling the NADP concentration to 2 mM during ferredoxin-mediated NADP reduction was found to slightly relieve the inhibition by heparin at low heparin concentrations, while reducing the NADP concentration to $0.5 \mathrm{~mm}$ was without effect (data not shown). This result suggests that high concentrations of NADP produce a weak effect on the binding of heparin to FNR under the conditions used to assay NADP reduction. The possible interaction of heparin with the pyridine nucleotide binding site on FNR can be elucidated in the absence of ferredoxin by examining

TABLE I

Effect of Heparin on Electron Transport to Anionic and Cationic PSi Acceptors

\begin{tabular}{cccccc}
\hline & \multicolumn{3}{c}{ Electron Transport } \\
\cline { 2 - 6 } $\begin{array}{c}\text { Heparin } \\
\mu \mathrm{M})\end{array}$ & FD/NADP & FD & FE $(\mathrm{CN})_{6}{ }^{3-}$ & AQS & MeV \\
\hline & & & & & \\
10 & 150 & 96 & 146 & 230 & 223 \\
100 & 77 & 90 & 136 & 207 & 222 \\
\hline
\end{tabular}

Note. Assay conditions are described under Materials and Methods. The concentrations of PSI acceptors were $10 \mu \mathrm{M}$ ferredoxin $/ 1 \mathrm{mM}$ NADP (plus 840 units of catalase) for NADP reduction, $21 \mu \mathrm{M}$ ferredoxin when used alone for ferredoxin-mediated oxygen reduction, $2.5 \mathrm{mM} \mathrm{Fe}(\mathrm{CN})_{6}{ }^{3}, 10 \mu \mathrm{M} \mathrm{AQS}$, and $67 \mu \mathrm{M} \mathrm{MeV}$. Electron transport rates are expressed as $\mu \mathrm{mol} \mathrm{O}_{2} \mathrm{~h}^{-1} \mathrm{mg} \mathrm{Chl}^{-1}$. 

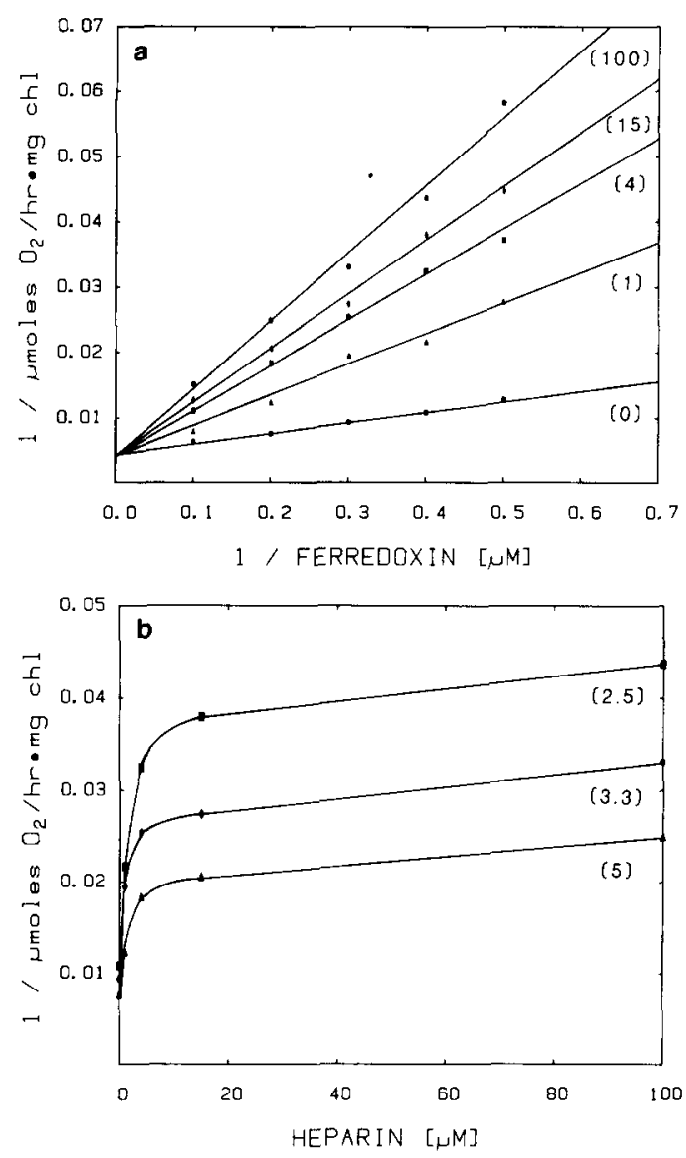

FIG. 2. Partial competitive kinctics of heparin inhibition of NADP reduction. (a) Double-reciprocal plots with ferredoxin as the varying substrate. Assay conditions are described under Materials and Methods. Values in parentheses represent the concentration $(\mu \mathrm{M})$ of heparin added to the reaction. (b) Dixon plots at various ferredoxin concentrations. Ferredoxin concentrations $(\mu \mathrm{M})$ are given in parentheses.

the effect of heparin on the diaphorase reaction (NADPH $\rightarrow$ FNR $\rightarrow$ DCPIP) under a variety of conditions.

The FNR-catalyzed reduction of DCPIP by NADPH at low ionic strength was inhibited by concentrations of heparin 100 -fold less than those required to inhibit ferredoxin-mediated NADP reduction (Fig. 3). As Fig. 3 also shows, increasing the NADPH concentration in the assay to $1 \mathrm{~mm}$ decreased the extent of heparin inhibition of the diaphorase reaction; at this NADPH concentration, a maximal inhibition of $23 \%$ was observed, in contrast

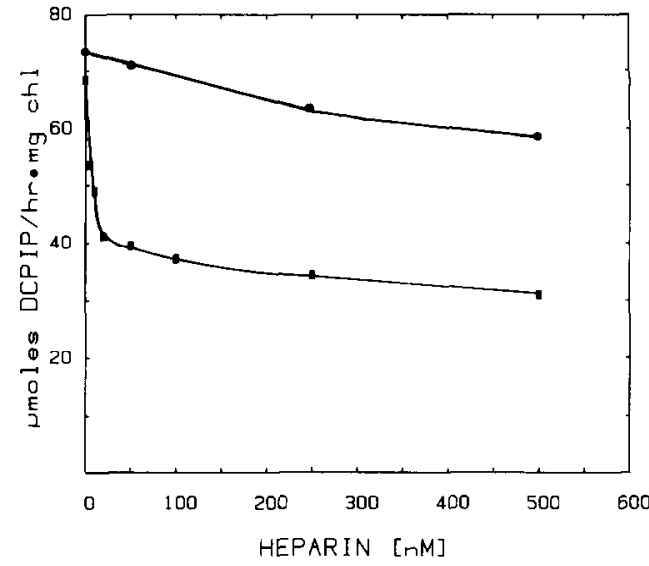

FIG. 3. Inhibition of the diaphorase reaction by heparin. Assays of DCPIP reduction catalyzed by membrane-bound FNR were performed as described under Materials and Methods. The reactions were initiated by the addition of NADPH to a final concentration of $40 \mu \mathrm{M}(\boldsymbol{C})$ or of $1 \mathrm{mM}(\Theta)$.

to the $56 \%$ inhibition obtained with $40 \mu \mathrm{M}$ NADPH as the substrate. Kinetic analysis of heparin inhibition of the diaphorase reaction showed a family of lines which converged on the $1 / v$ axis (Fig. 4). Dixon plots were not presented, but could be seen by inspection of Fig. 4 to have been

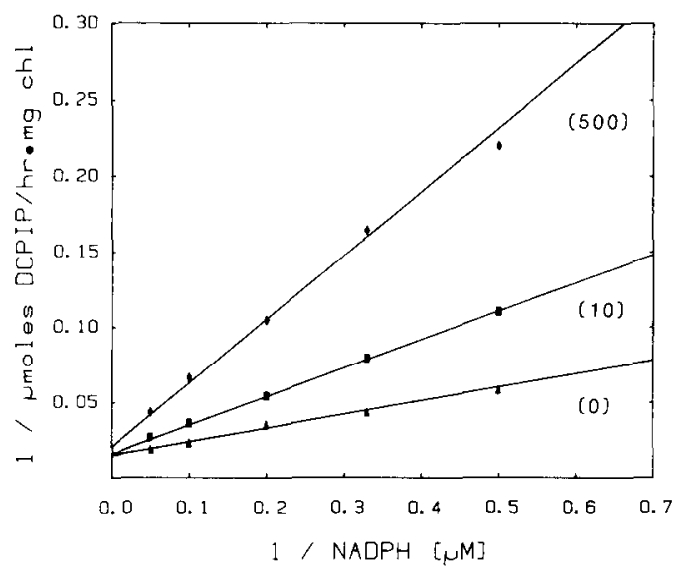

FIG. 4. Partial competitive kinetics of heparin inhibition of the diaphorase reaction. Double- reciprocal plots with $\mathrm{N} \Lambda \mathrm{DPH}$ as the varying substrate are presented. Assay conditions are given under Materials and Methods. Values in parentheses represent the concentration ( $\mathrm{nM}$ ) of heparin added to the reaction. 
nonlinear. Therefore, heparin was partially competitive with respect to NADPH in the diaphorase reaction, and partially competitive with respect to ferredoxin during NADP reduction. The $K_{m}$ for NADPH in the diaphorase reaction was measured to be $6.3 \mu \mathrm{M}$, while the $K_{i}$ of the heparin:FNR association (determined as above) was $5 \mathrm{nM}$.

Ferredoxin by itself is partially competitive with respect to NADPH during assays of DCPIP reduction at low ionic strength (19); the inhibition is reversed by the addition of $\mathrm{NaCl}$. Similarly, heparin inhibition of the diaphorase reaction can be largely relieved by an increase in the ionic strength of the medium. In the presence of $80 \mathrm{mM} \mathrm{NaCl}$ or greater, the rate of DCPIP reduction was approximately $75 \%$ that of uninhibited reaction, and the $K_{i}$ of the heparin:FNR association increased with ionic strength from $5 \mathrm{~nm}$ at $20 \mathrm{mM} \mathrm{NaCl}$, to $1 \mu \mathrm{M}$ at $150 \mathrm{mM} \mathrm{NaCl}$ (data not shown). This decrease in heparin inhibition with increasing ionic strength is probably due to the dissociation of a heparin:FNR complex stabilized by ionic interactions.

\section{DISCUSSION}

Micromolar concentrations of heparin have little effect on the reduction of ferredoxin by PSI, but effectively inhibit ferredoxin-mediated NADP reduction by inhibiting ferredoxin binding to FNR in a partially competitive manner. The FNRcatalyzed reduction of DCPIP by NADPH is inhibited by heparin in a similar manner. In the latter case the inhibition is largely, but not completely, relieved by increasing the ionic strength of the medium or by increasing the concentration of NADPH. While heparin inhibits the binding of both ferredoxin and pyridine nucleotides to FNR, under the conditions used to measure NADP reduction (an ionic strength of $120 \mathrm{mM}$ and a pyridine nucleotide concentration of $1 \mathrm{~mm}$ ), the major effect involves interference with ferredoxin binding to FNR. Likewise, the experiments involving heparin inhibition of the diaphorase reaction reveal a number of similarities between the interaction of heparin and of ferredoxin with FNR. Like heparin, ferredoxin competes partially with NADPH for binding to FNR at low ionic strength $(I=10 \mathrm{mM})(19)$. Both ferredoxin and heparin are more tightly bound to FNR under low salt conditions, as evidenced by their similar $K_{i}$ values, for inhibition of the diaphorase reaction [ $5 \mathrm{~nm}$ for heparin vs $1.9 \mathrm{~nm}$ for ferredoxin (19)]. At an intermediate ionic strength ( $I-50-100 \mathrm{~mm}$ ) ferredoxin stimulates the diaphorase reaction $(19,20)$, apparently by enhancing the association of DCPIP with FNR. The degree of stimulation declines with increasing ionic strength, reflecting both the dissociation of the ferredoxin:FNR complex (20) and the inactivation of FNR by high salt concentrations (19). We have observed that the inhibitory heparin:FNR complex dissociates with increasing ionic strength $\left(K_{i}\right.$ increases from $5 \mathrm{nM}$ to $1 \mu \mathrm{M}$ ), indicating that this complex, like that of ferredoxin:FNR, depends primarily upon ionic interactions. While both heparin and ferredoxin form ionic complexes with FNR which inhibit the diaphorase reaction, heparin fails to stimulate the reaction at higher ionic strengths.

The data obtained for the diaphorase reaction initiated by $1 \mathrm{~mm}$ NADPH (Fig. 3 ) are consistent with an increased $K_{i}$ for heparin in the presence of high concentrations of pyridine nucleotide. This decreased association of heparin with FNR can be compared to earlier kinetic studies which show that both NADPH and NADP inhibit ferredoxin binding to FNR (21). These results can also be compared to a recent report that $0.5 \mathrm{~mm}$ NADP increases the $K_{d}$ of the ferredoxin:FNR complex at least 14-fold (22).

The kinetic analyses presented in this paper indicate that heparin does not completely prevent the binding of ferredoxin or NADPH to FNR, but rather increases the $K_{s}$ for both substrates. This type of behavior could arise from heparin binding at a site or sites which overlap the ferredoxin and pyridine nucleotide binding sites on FNR, or from binding at a site which is distinct from these sites, but 
from which heparin can affect ligand binding. While our data do not identify the site of heparin binding, an estimation of the number of heparin binding sites on FNR can be made from the data of Fig. 3. Heparin is tightly bound to FNR at low ionic strength, and under these conditions the inhibition pattern is clearly biphasic with increasing heparin concentration. The intersection of tangents to the two slopes falls at $20 \mathrm{~nm}$ heparin. This concentration of the polysaccharide accounts for $73 \%$ of the total inhibition seen using 500 $\mathrm{nM}$ heparin. The concentration of FNR present in the experiment can be estimated on the basis of chlorophyll concentration using the results in (23). When this was done, the ratio of heparin to FNR at $20 \mathrm{~nm}$ heparin was found to be 1:3.5. Heparin may be acting to aggregate membrane-bound FNR; in any event no more than one heparin binding site per FNR is indicated.

A few comments are in order concerning the use of heparin as an inhibitor in photosynthetic experiments. Ferredoxinmediated NADP reduction can be inhibited to a maximum of $34 \%$ of control; while the inhibition by heparin is not complete, it is as effective as the more laborious inactivation of FNR with $N$ ethylmaleimide. Heparin is active at a 1000-fold lower concentration than pyrophosphate, and therefore contributes little to the ionic character of the medium. The components of the electron transport chain prior to FNR are unaffected by the polysaccharide. Likewise, photophosphorylation is not affected by heparin; we have found that the $\mathrm{P} / 2 \mathrm{e}$ of noncyclic electron transport to methylviologen remains the same (1.25) in the presence of 10 or 100 $\mu \mathrm{M}$ heparin.

\section{ACKNOWLEDGMENTS}

This research was supported by a grant (PCM8214240) from the National Science Foundation.
J.P.H. received predoctoral support from a DOE fellowship program administered by The University of Michigan Office of Energy Research.

\section{REFERENCES}

1. Keister, D. L., SAN Pietro, A., ANd Stolzenbach, F. E. (1960) J. Biol. Chem. 235, 2989-2996.

2. Shin, M., Tagawa, K., and ARnon, D. I. (1963) Biochem. Z. 338, 84-96.

3. Davis, D. J., ANd SAN Pietro, A. (1977) Biochem. Biophys. Res. Commun. 74, 33-40.

4. Avron, M., AND Jagendorf, A. (1956) Arch. Biochem. Biophys. 65, 475-490.

5. Avron, M., And Jagendorf, A. (1957) Arch. Biochem. Biophys. 72, 17-24.

6. Boger, P. (1979) Encycl Plant Physiol New Ser. 6, 399-409.

7. Neldsun, N., and Ngumann, J. (1968) Biochem. Biophys. Res. Commun. 30, 142-147.

8. Shin, M., and San Pietro, A. (1968) Biochem. Biophys. Res. Commun. 33, 38-42.

9. Foust, G. P., Mayhew, S. G., and Massey, V. (1969) J. Biol Chem. 244, 964-970.

10. Forti, G., AND Meyer, E. M. (1969) Plant Physiol 44, 1511-1514.

11. Helbert, J. R., and Marini, M. A. (1963) Biochemistry 5, 1101.

12. Sumyk, G. B., AND Yocum, C. F. (1968) J. Chromatogr. 35, 101-103.

13. Robinson, H. H., AND Yocum, C. F. (1980) Biochim. Biophys. Acta 590, 97-106.

14. Petering, D. H., and Palmer, G. (1970) Arch. Biochem. Biophys. 141, 456-464.

15. Yocum, C. F. (1982) in Methods in Chloroplast Molecular Biology (Edelman, M., Hallick, R. B., and Chua, N.-H., eds.), pp. 973-981, Elsevier, Amsterdam/New York.

16. Arnon, D. I. (1949) Plant Physiol 24, 1-15.

17. Dixon, M., AND WebB, E. C. (1964) Enzymes, p. 320, Longman, Green and Co., London.

18. SegEL, I. H. (1975) Enzyme Kinetics, p. 184, Wiley, New York.

19. Nakamura, S., AND Kimura, T. (1971) J. Biol. Chem. 246, 6235-6241.

20. BöGer, P. (1971) Planta (Berlin) 99, 319-338.

21. Nelson, N., and Neumann, J. (1969) J. Biol. Chem. 244, 1926-1931.

22. Batie, C. J. (1983) Ph.D. Thesis, Duke University. 23. Вӧнме, H. (1978) Eur. J. Biochem. 83, 137-141. 